\title{
POTENTIAL IR OBSERVATIONS OF THE SOLAR CORONA
}

\author{
C. KRISHNA KUMAR \\ Department of Astronomy, Howard University, Washington, DC 20059, U.S.A. \\ and \\ JOSEPH DAVILA \\ Code 682, NASA/Goddard Space Flight Center, Greenbelt, MD 20771, U.S.A.
}

\begin{abstract}
We examine the potential for observations of the solar corona using forbidden lines in the near infrared.

Key words: infrared: stars - Sun: corona
\end{abstract}

\section{Introduction}

Observations of IR emission lines beyond $1 \mu \mathrm{m}$ wavelength in the spectrum of the corona were first made during eclipses in the 1960's by Mangus and Stockhausen (1966) and by Münch et al. (1967). There have been no reports of subsequent investigations of the IR spectrum of the corona. In view of the developments in IR technology a re-examination of the potential IR studies of the corona was felt worthwhile. The sources of IR lines in the coronal spectrum and the issues involved in studying them are discussed below, followed by a section on the possible contributions to coronal physics and other fields.

There are two sources of IR lines. They are: (i) the excited fine structure levels of the ions in the coronal material, and (ii) fine structure levels of atoms and ions of the material shed by sun-grazing comets. The comet source is available infrequently (perhaps 1 or 2 days a year) but it offers possibly the only opportunity for measuring coronal magnetic fields (see discussion in last section).

\section{Sources of IR Lines}

\subsection{Coronal Material}

In the corona proper, above the transition region, highly charged ions with ionization energies near $500 \mathrm{eV}$ dominate the ionization equilibrium. Most of these ions do not have fine structure levels in the ground state that can emit in the IR. One exception is the $1.43 \mu \mathrm{m}$ line of $\mathrm{Si} \mathrm{X}$. Cosmically underabundant elements, $\log (X / H)<-7.0$, may have appropriate levels but they are not considered here. In the transition region the lower ionization stages can emit many IR lines.

\subsection{Sungrazing Comets}

Sungrazing comets pass through the corona and shed neutral gases and dust particles. The dust particles evaporate to produce atoms which along with the gas phase atoms are ionized to higher charge states until they reach ionization equilibrium with the coronal matter. This process can take hours to days before equilibrium is reached. Therefore the comet material will exhibit all the ionization stages seen 
in the transition region except that this will happen in the corona. These lowerionization stages can result in a rich IR line spectrum. Table I lists the ions of the cosmically more abundant species expected to be in comets which will emit IR lines from their ground state fine structure levels.

There are two type of sungrazing comets. They are: (i) the bright Ikeya-Seki (1965) which appear very seldom (every 50 years or so) and, (ii) the dimmer pygmies which averaged one a year over the last decade. These pygmies were discovered (Sheely et al.1982, MacQueen and St. Cyr 1991) using the coronographs on SOLWIND and SMM satellites. They have perihelion distances $q<5 R_{\odot}$ with many falling into the sun. None of the sungrazers discovered on SMM images were found on groundbased coronograph pictures (MacQueen and St. Cyr 1991). Their peak surface brightness is $2 \times 10^{-8} B_{\odot}$ at $530 \mathrm{~nm}$. This is two orders of magnitude lower than the detection limits of existing groundbased coronographs operating in the visual. However in the case of SOLWND-1, the brightest known pygmy sungrazer, a post-perihelion enhancement of the corona was detected by the SOLWIND coronograph. This appears to have been detected in a ground based spectrum also, taken using a coronagraph (Chocol et al. 1983). The observers claim to have detected lines of Si II and Ni II. Therefore it may be possible in some bright cases to observe the spectra of sungrazers from the ground if one knows where to look for them. In the IR at least an order of magnitude decrease in the level of the atmospherically scattered light is possible (in units of $B_{\odot}$ ). If, in addition, the coronagraph is located on an aircraft the sky brightness drops to $10^{-7} B_{\odot}$ at least (Newkirk and Eddy 1964). This makes the spectroscopy of sungrazing comets, using an aircraft based coronagraph, especially attractive. Whether the cometary lines can be detected depends on the unknown mass loss rate of the comet. The reported detection by Chocol et al. (1983) of the cometary lines is encouraging. Attempts should be made to observe the spectra of the sungrazing comets. To do this one needs to find the comet first.

\section{Observational Constraints}

If space-based coronagraphs continue to be the sole means for discovering sungrazing pygmy comets one has at most a few hours or a day before they fall into the sun or go outside $10 R_{\odot}$. This is because the coronagraph's field of view is limited to say $10 R_{\odot}$ centered on the sun. Real time information is needed to execute spectroscopic observations from the ground or from aircraft equipped with coronagraphs. Such real time detection of the comets was not possible with the SOLWIND and SMM instruments, but it may be with the proposed LASCO coronagraph. This instrument has an outer limit of $30 R_{\odot}$ and can give 2 or 3 day's notice so that even balloon-based observations can be made.

Münch et al. (1967) measured radiance of the purely coronal line at $1.43 \mu \mathrm{m}$ to be about $10^{-4} B_{\odot}$ along a path $0.2 R_{\odot}$ above the limb. It should be possible to measure the $\mathrm{Si} \mathrm{X}$ line, out to $3 R_{\odot}$, from the ground and further out from an aircraft. In this connection it is worth noting that the measured sky brightness at $2.2 \mu \mathrm{m}$ from a balloon coronagraph was $10^{-12} B_{\odot}$ (MacQueen, 1968).

In summary, the Si X line at $1.43 \mu \mathrm{m}$ is probably bright enough to use for map- 
ping the corona out to $3 R_{\odot}$ using a ground-based coronagraph and IR imager or more likely with an aircraft mounted system. A balloon-based system will definitely extend the coverage to greater distances from the sun's limb. Spectroscopic observations of sungrazing comets may also be possible. To do this real time detection of the comet from space based coronagraphs is necessary until other methods of discovering them are developed.

\section{Scientific Expectations}

Finally, what are the scientific reasons for the IR observations of comets?

- The possibility that IR coronagraphs may have lower scattered light background than the visual band instruments, makes imaging of the corona using the $1.43 \mu \mathrm{m}$ light of Si X attractive. It may extend the observable distance from the limb beyond that possible with the green line. It will, at least, supplement the observations in the visual.

- The ions expected to appear in the spectra of sungrazing comets cover a large range in ionization energies, from a few $\mathrm{eV}$ to $500 \mathrm{eV}$. The relative concentrations of the ions can yield information about ionization rates in different parts of the corona.

- The spectra of the sungrazers can be used to derive the chemical abundances of cometary material, including the non-volatile elements. Until the Halley fly-by, such information was not available.

- $\quad$ One of the ions expected in the cometary debris is Ca II. The upper levels emitting the triplet near $850 \mathrm{~nm}$ will be excited by sunlight. The lifetime of this level is appropriate for measuring the magnetic fields in the $0.1 \mathrm{G}$ to $1 \mathrm{G}$ by means of the Hanle effect. This may be the only spectroscopic method for measuring coronal magnetic fields.

TABLE I

IR line-emitting ions in the spectra of sungrazing comets

\begin{tabular}{cc}
\hline Emitting in 1-10 $\mu$ m range & Emitting in 10-100 $\mu \mathrm{m}$ range \\
\hline Mg IV,V,VII,VIII & N III \\
Si VI,VII,IX,X & O III,IV \\
Ca IV,V,VII,VIII & Ti IV \\
Ti VI,VII & Cr III,IV,V,VI \\
Cr V & Fe V,VI \\
Fe VII,VIII & Ni VII \\
Ni VII,VIII & \\
\hline
\end{tabular}




\section{References}

Chocol, D., Rušin, V., Kulcar, L., and Vanysek, V.: 1983, Astrophys. and Sp. Sci. 91, 71. Macqueen, R.M.: 1968, Ap.J. 154, 1062.

Macqueen, R.M., and St. Cyr, O.C.: 1991, Icarus 90, 96.

Mangus, J., and Stockhausen, R.: 1966, NASA Rept. X-614-66-29.

Münch, G., Neugebauer, G., and McCammon, D.: 1967 Astrophys. J. 149, 681.

Sheeley, N.R., Howard, R.A., Koomen, M.J., and Michels, D.J.: 1982, Nature, 300, 239.

Newkirk, G. and Eddy, J.: 1964 J. Atmos. Phys., 21, 34. 